PROCEEDINGS OF THE

AMERICAN MATHEMATICAL SOCIETY

Volume 130, Number 10, Pages 2941-2946

S 0002-9939(02)06456-0

Article electronically published on March 13, 2002

\title{
EXCESSES, DUALITY GAPS AND WEAK COMPACTNESS
}

\author{
STEPHEN SIMONS
}

(Communicated by Jonathan M. Borwein)

\begin{abstract}
We explore the connection between the concepts "excess" and "duality gap" from epigraphical analysis and optimization, and the functional analytic concepts of weak* and weak compactness. We also discuss briefly the connection with R. C. James's "sup theorem".
\end{abstract}

\section{Introduction}

In this paper, we will explore the connection between two concepts from epigraphical analysis and optimization, "excess" and "duality gap", and the classical functional analytic concepts of weak* and weak compactness.

If $\widetilde{X}$ and $X$ are subsets of a normed space, then the excess of $\widetilde{X}$ over $X$ is defined by

$$
e(\widetilde{X}, X):=\sup _{z \in \widetilde{X}} \operatorname{dist}(z, X)=\sup _{z \in \widetilde{X}} \inf _{y \in X}\|z-y\| .
$$

See Beer [1, §1.5, pp. 28-33] for more information on this concept.

We will use the concept of duality gap only with reference to the canonical bilinear form associated with a normed space and its dual - we will define it at the beginning of Section 1 below.

The main result on excess and duality gap appears in Theorem 2(a), and it leads rapidly to Theorem 2(b), a minimax criterion for certain subsets of a dual space to be weak* compact. Corollary 4 contains an application of Theorem 2(b) to reflexive spaces.

One of the side-effects of the Banach-Alaoglu theorem is that weak* compactness is much less interesting than weak compactness, and so we show in Theorem 5 how to adapt Theorem 2 to obtain results on weak compactness. The bidual is mentioned in Theorem 5(a), however it is interesting and significant that Theorem 5 (b) is bidual-free.

Theorem 5(b) is an old result, which was first obtained in connection with R. C. James's sup theorem. We discuss this issue briefly in Section 3.

We shall use the following classical minimax theorem frequently as a computational tool. It follows from a result of Fan; see [2] or [4 Theorem 3.1, p. 17].

Received by the editors May 1, 2001.

2000 Mathematics Subject Classification. Primary 46B10, 46N10, 49J35, 49 N15.

Key words and phrases. Normed space, excess, minimax theorem, duality gap, weak-star and weak compactness. 
Lemma 1. Let $X$ be a nonempty convex subset of a vector space, $Y$ be a nonempty convex subset of a vector space and $Y$ also be a compact Hausdorff topological space. Let $f: X \times Y \mapsto \mathbb{R}$ be convex on $X$, and concave and upper semicontinuous on $Y$. Then

$$
\inf _{X} \max _{Y} f=\max _{Y} \inf _{X} f
$$

\section{The BASIC RESUlt ON EXCESS AND DUALITY GAP}

In this section, $F$ is a real normed space with dual $F^{*}$ and unit ball $B$. $\mathcal{C}$ stands for the set of all nonempty convex subsets of $B, \mathcal{C C}$ stands for the set of all nonempty closed convex subsets of $B$, and $\langle\cdot, \cdot\rangle$ is the canonical bilinear form on $F \times F^{*}$. We shall use $\delta$ as an alias for $\langle\cdot, \cdot\rangle$, so if $Y$ is a nonempty convex subset of $F$ and $X$ is a nonempty convex subset of $F^{*}$, we write "sup $\inf _{Y} \delta$ " for $\sup _{x^{*} \in X} \inf _{y \in Y}\left\langle y, x^{*}\right\rangle$ and "inf $\sup _{X} \delta$ " for $\inf _{y \in Y} \sup _{x^{*} \in X}\left\langle y, x^{*}\right\rangle$. Then, as is well known, $\sup _{X} \inf _{Y} \delta \leq \inf _{Y} \sup _{X} \delta$. We shall write

$$
\operatorname{dgap}(Y, X):=\inf _{Y} \sup _{X} \delta-\sup _{X} \inf _{Y} \delta .
$$

"dgap" stands for "duality gap". However, we should caution the reader that some authors use the phrase "duality gap" for the interval $\left[\sup _{X} \inf _{Y} \delta, \inf _{Y} \sup _{X} \delta\right]$.

Theorem 2. (a) Let $X$ be a nonempty bounded convex subset of $F^{*}$ and $\tilde{X}$ be the $w\left(F^{*}, F\right)$-closure of $X$ in $F^{*}$. Then

$$
\begin{aligned}
e(\tilde{X}, X) & =\sup _{Y \in \mathcal{C}} \operatorname{dgap}(Y, X) \\
& =\sup _{Y \in \mathcal{C} \mathcal{C}} \operatorname{dgap}(Y, X) .
\end{aligned}
$$

(b) Let $\bar{X}$ be the norm-closure of $X$. Then the conditions (2.3)-(2.5) are equivalent:

$$
\begin{aligned}
& \qquad \bar{X} \text { is } w\left(F^{*}, F\right)-\text { compact. } \\
& \text { For all } Y \in \mathcal{C}, \quad \inf _{Y} \sup _{X} \delta=\sup _{X} \inf _{Y} \delta \\
& \text { For all } Y \in \mathcal{C C}, \quad \inf _{Y} \sup _{X} \delta=\sup _{X} \inf _{Y} \delta
\end{aligned}
$$

Proof. (a) The Banach-Alaoglu theorem implies that $\widetilde{X}$ is $w\left(F^{*}, F\right)$-compact. Further, it is clear that

$$
y \in F \quad \Longrightarrow \quad \max \langle y, \widetilde{X}\rangle=\sup \langle y, X\rangle \text {. }
$$

We now prove the inequality " $\geq$ " in (2.1). To this end, let $Y \in \mathcal{C}, y \in Y, z^{*} \in \widetilde{X}$ and $x^{*} \in X$. Then, since $y \in B$,

$$
\left\langle y, z^{*}\right\rangle=\left\langle y, x^{*}\right\rangle+\left\langle y, z^{*}-x^{*}\right\rangle \leq\left\langle y, x^{*}\right\rangle+\left\|z^{*}-x^{*}\right\| .
$$

We first take the infimum over $y \in Y$ and reorder the terms and derive that

$$
\inf \left\langle Y, z^{*}\right\rangle-\inf \left\langle Y, x^{*}\right\rangle \leq\left\|z^{*}-x^{*}\right\| \text {. }
$$

Taking the infimum over $x^{*} \in X$, we obtain

$$
\inf \left\langle Y, z^{*}\right\rangle-\sup _{X} \inf _{Y} \delta \leq \operatorname{dist}\left(z^{*}, X\right) .
$$

Finally, taking the supremum over $z^{*} \in \tilde{X}$, we obtain

$$
\sup _{\widetilde{X}} \inf _{Y} \delta-\sup _{X} \inf _{Y} \delta \leq e(\widetilde{X}, X) .
$$


From Lemma 1 and (2.6),

$$
\sup _{\widetilde{X}} \inf _{Y} \delta=\inf _{Y} \sup _{\widetilde{X}} \delta=\inf _{Y} \sup _{X} \delta .
$$

Substituting this into (2.7) gives

$$
\inf _{Y} \sup _{X} \delta-\sup _{X} \inf _{Y} \delta \leq e(\widetilde{X}, X) .
$$

That is to say, $\operatorname{dgap}(Y, X) \leq e(\tilde{X}, X)$. Since this holds for all $Y \in \mathcal{C}$, we can take the supremum of the left-hand side and obtain the inequality " $\geq$ " in (2.1). The inequality " $\geq$ " in (2.2) is immediate, so it only remains for (a) to prove that

$$
\sup _{Y \in \mathcal{C C}} \operatorname{dgap}(Y, X) \geq e(\widetilde{X}, X) .
$$

There is so much "loss" in the the proof of the inequality " $\geq$ " in (2.1) above that it seems unlikely that (2.8) holds. However, it does, and here is a proof. Let

$$
\alpha:=\sup _{Y \in \mathcal{C C}} \operatorname{dgap}(Y, X) .
$$

Then

$$
Y \in \mathcal{C C} \quad \Longrightarrow \quad \operatorname{dgap}(Y, X) \leq \alpha .
$$

Let $z^{*} \in \widetilde{X}$ and $\varepsilon>0$. Since $\left\langle B, z^{*}\right\rangle$ is bounded and $z^{*}$ is continuous and linear on $F$, we can write $B=Y_{1} \cup \cdots \cup Y_{m}$, where, for all $i \in\{1, \ldots, m\}$,

$$
Y_{i} \in \mathcal{C C} \quad \text { and } \sup \left\langle Y_{i}, z^{*}\right\rangle \leq \inf \left\langle Y_{i}, z^{*}\right\rangle+\varepsilon \text {. }
$$

Combining this with (2.6), we obtain

$$
\sup \left\langle Y_{i}, z^{*}\right\rangle \leq \inf _{Y_{i}} \sup _{X} \delta+\varepsilon
$$

Further, from the definition of "supremum", for all $i \in\{1, \ldots, m\}$ there exists $x_{i}^{*} \in X$ such that

$$
\inf \left\langle Y_{i}, x_{i}^{*}\right\rangle \geq \sup _{X} \inf _{Y_{i}} \delta-\varepsilon
$$

Let $K$ be the convex hull of the finite set $\left\{x_{1}^{*}, \ldots, x_{m}^{*}\right\} . K$ is a norm-compact convex subset of $X$. We shall prove that

$$
\operatorname{dist}\left(z^{*}, K\right) \leq \alpha+2 \varepsilon
$$

Once this has been done, it is evident that $\operatorname{dist}\left(z^{*}, X\right) \leq \alpha+2 \varepsilon$. Since this construction can be carried out for all $z^{*} \in \widetilde{X}$, and $\varepsilon$ can be made arbitrarily small, $e(\widetilde{X}, X) \leq \alpha$, and (2.8) follows, as required. In order to establish (2.12), we note from Lemma 1 that

$$
\begin{aligned}
\operatorname{dist}\left(z^{*}, K\right) & :=\min _{x^{*} \in K}\left\|z^{*}-x^{*}\right\| \\
& =\min _{x^{*} \in K} \sup _{y \in B}\left\langle y, z^{*}-x^{*}\right\rangle=\sup _{y \in B} \min _{x^{*} \in K}\left\langle y, z^{*}-x^{*}\right\rangle .
\end{aligned}
$$

Since $B=Y_{1} \cup \cdots \cup Y_{m}$ and each $x_{i}^{*} \in K$, we derive that

$$
\begin{aligned}
\operatorname{dist}\left(z^{*}, K\right) & =\max _{i=1}^{m} \sup _{y \in Y_{i}} \min _{x^{*} \in K}\left\langle y, z^{*}-x^{*}\right\rangle \\
& \leq \max _{i=1}^{m} \sup _{y \in Y_{i}}\left\langle y, z^{*}-x_{i}^{*}\right\rangle \\
& \leq \max _{i=1}^{m}\left[\sup \left\langle Y_{i}, z^{*}\right\rangle-\inf \left\langle Y_{i}, x_{i}^{*}\right\rangle\right] .
\end{aligned}
$$

Consequently, using (2.10) and (2.11),

$$
\begin{aligned}
\operatorname{dist}\left(z^{*}, K\right) & \leq \max _{i=1}^{m}\left[\inf _{Y_{i}} \sup _{X} \delta-\sup _{X} \inf _{Y_{i}} \delta+2 \varepsilon\right] \\
& =\max _{i=1}^{m}\left[\operatorname{dgap}\left(Y_{i}, X\right)+2 \varepsilon\right] .
\end{aligned}
$$


Since each $Y_{i} \in \mathcal{C C}$, we derive from (2.9) that

$$
\operatorname{dist}\left(z^{*}, K\right) \leq \max _{i=1}^{m}[\alpha+2 \varepsilon]=\alpha+2 \varepsilon .
$$

This gives (2.12), and completes the proof of (a).

(b) We first prove that (2.3) is equivalent to

$$
\bar{X} \supset \tilde{X} \text {. }
$$

Indeed, if (2.3) is satisfied, then $\bar{X}$ is $w\left(F^{*}, F\right)$-closed and (2.13) follows immediately. If, conversely, (2.13) is satisfied, then, since $\widetilde{X} \supset \bar{X}$ in any case, $\widetilde{X}=\bar{X}$ and the Banach-Alaoglu theorem gives (2.3). Thus (2.3) and (2.13) are equivalent. However, $(2.13)$ is equivalent to the statement that $e(\widetilde{X}, X)=0$, and the equivalence of (2.13) with (2.4) and (2.5) follows from (a).

Remark. Of course, by performing an appropriate scaling, we can obtain two further equivalent conditions in Theorem 2 (b) by replacing $\mathcal{C}$ in $(2.4)$ by the set of all nonempty bounded convex subsets of $F$ (instead of $B$ ) and replacing $\mathcal{C C}$ in $(2.5)$ by the set of all nonempty bounded closed convex subsets of $F$.

Corollary 3. Let $Y$ be a nonempty bounded convex subset of $F$ and $X$ be a nonempty convex subset of $F^{*}$ such that the $w\left(F^{*}, F^{* *}\right)$-closure of $X$ is $w\left(F^{*}, F\right)$ compact. Then

$$
\inf _{Y} \sup _{X} \delta=\sup _{X} \inf _{Y} \delta
$$

Proof. Since $X$ is convex, its norm-closure is identical with its $w\left(F^{*}, F^{* *}\right)$-closure. The result now follows from Theorem 2(b), enhanced as in the remark above.

The author is grateful to both the referee and Professor Jon Borwein for pointing out that the following result is well-known, and can be proved in several other ways.

Corollary 4. Let $F$ be a reflexive Banach space, $Y$ be a nonempty bounded convex subset of $F$ and $X$ be a nonempty bounded convex subset of $F^{*}$. Then

$$
\inf _{Y} \sup _{X} \delta=\sup _{X} \inf _{Y} \delta .
$$

Proof. This follows from Corollary 3 , since the reflexivity of $F$ implies $w\left(F^{*}, F^{* *}\right)=$ $w\left(F^{*}, F\right)$.

Remark. It follows from Lemma 1 that if $F$ is a reflexive Banach space, $Y$ is a (possibly unbounded) nonempty convex subset of $F$ and $X$ is a nonempty bounded closed convex subset of $F^{*}$, then (3.1) holds. It is tempting to surmise that (3.1) holds if $Y$ is a nonempty closed convex subset of $F$ and $X$ is a nonempty bounded convex subset of $F^{*}$ (we are transferring the "closedness" from $X$ to $Y$ - another way of looking at this is an attempted "interpolation" between Lemma 1 and Corollary 4). However, this is false, as can be seen from the following simple example. Let $F:=\mathbb{R}, Y:=[0, \infty)$ and $X:=(-1,0)$. Then $\inf _{y \in Y} \sup _{x^{*} \in X} y x^{*}=0$ and $\sup _{x^{*} \in X} \inf _{y \in Y} y x^{*}=-\infty$.

\section{The DUAl SETUP, AND WEAK COMPACTNESS IN A NORMED SPACE}

In this section, we show how the results of Section 1 can be parlayed into results on weak compactness. $E$ is a real normed space, $\mathcal{C}$ stands for the set of all nonempty convex subsets of the unit ball of $E^{*}, \mathcal{C C}$ stands for the set of all nonempty normclosed convex subsets of the unit ball of $E^{*}$ and $\delta$ is the canonical bilinear form 
on $E \times E^{*}$. We point out again, as we have already done in the Introduction, the significant fact that the bidual of $E$ is not mentioned in Theorem 5(b) below.

Theorem 5. (a) Let $X$ be a nonempty bounded convex subset of $E, Z$ be the canonical image of $X$ in $E^{* *}$ and $\widetilde{Z}$ be the $w\left(E^{* *}, E^{*}\right)$-closure of $Z$ in $E^{* *}$. Then

$$
e(\widetilde{Z}, Z)=\sup _{Y \in \mathcal{C}} \operatorname{dgap}(Y, X)=\sup _{Y \in \mathcal{C C}} \operatorname{dgap}(Y, X) .
$$

(b) If, further, $X$ is norm-complete, then the conditions (5.1)-(5.3) are equivalent:

$$
\begin{aligned}
& \qquad \text { is } w\left(E, E^{*}\right) \text {-compact. } \\
& \text { For all } Y \in \mathcal{C}, \quad \inf _{Y} \sup _{X} \delta=\sup _{X} \inf _{Y} \delta \\
& \text { For all } Y \in \mathcal{C C}, \quad \inf _{Y} \sup _{X} \delta=\sup _{X} \inf _{Y} \delta .
\end{aligned}
$$

Proof. (a) This is immediate from Theorem 2(a) with $F:=E^{*}$.

(b) Since the canonical map is a $w\left(E, E^{*}\right)-w\left(E^{* *}, E^{*}\right)$ homeomorphism from $X$ onto $Z,(5.1)$ is equivalent to

$$
Z \text { is } w\left(E^{* *}, E^{*}\right) \text {-compact. }
$$

However, the canonical map is also a norm-isometry from $X$ onto $Z$, hence $Z$ is complete and so $Z=\bar{Z}$, the norm-closure of $Z$ in $E^{* *}$. Thus (5.4) is equivalent to

$$
\bar{Z} \text { is } w\left(E^{* *}, E^{*}\right) \text {-compact. }
$$

(b) now follows from Theorem 2(b), with $F:=E^{*}$.

Remark. As was the case with Theorem 2(b), by performing an appropriate scaling, we can obtain two further equivalent conditions in Theorem 5 (b) by replacing $\mathcal{C}$ in (5.2) by the set of all nonempty bounded convex subsets of $E^{*}$, and replacing $\mathcal{C C}$ in (5.3) by the set of all nonempty bounded closed convex subsets of $E^{*}$.

\section{Comments on James's theorem}

The equivalence of (5.1) and (5.2) was first proved in [3, Theorem 15, p. 717]. The following result was also proved in [3, Theorem 14, pp. 715-6]:

Theorem 6. If $X$ is a nonempty bounded convex (not necessarily closed) subset of $E$ and each element of $E^{*}$ attains its supremum on $X$, then (5.2) holds.

It is clear that if we combine Theorem 6 and Theorem 5 , we can obtain a proof of:

James's Theorem. If $X$ is a nonempty bounded convex norm-complete subset of $E$ and each element of $E^{*}$ attains its supremum on $X$, then $X$ is $w\left(E, E^{*}\right)$-compact.

The proof of Theorem 6 given in 3 is rather hard. It is possible to give a much simpler proof of Theorem 6 for locally convex spaces that, when specialized to normed spaces, gives the following result: If $X$ is a nonempty bounded convex (not necessarily closed) subset of $E$ and there exists $\psi \in \ell_{\infty}(X)$ such that, for all $x^{*} \in E^{*},\left.x^{*}\right|_{X}-\psi$ attains its supremum on $X$, then (5.2) holds. The corresponding generalization of James's theorem is then: If $X$ is a nonempty bounded convex norm-complete subset of $E$ and there exists $\psi \in \ell_{\infty}(X)$ such that, for all $x^{*} \in E^{*}$, $\left.x^{*}\right|_{X}-\psi$ attains its supremum on $X$, then $X$ is $w\left(E, E^{*}\right)$-compact. Details of these results will appear elsewhere. 


\section{ACKNOWLEDGEMENT}

The author would like to acknowledge several useful conversations with Manuel Ruiz Galán during the initial work on this paper.

\section{REFERENCES}

1. G. Beer, Topologies on closed and closed convex sets, Mathematics and Its Applications 268 (1993). MR 95k:49001

2. K. Fan, Minimax theorems, Proc. Nat. Acad. Sci. U.S.A. 39 (1953), 42-47. MR 14:1109f

3. S. Simons, Maximinimax, minimax, and antiminimax theorems and a result of $R$. C. James, Pac. J. Math. 40 (1972), 709-718. MR 47:756

4. Minimax and monotonicity, Lecture Notes in Mathematics 1693 (1998). MR 2001h: 49002

Department of Mathematics, University of California, Santa Barbara, California 93106-3080

E-mail address: simons@math.ucsb.edu 